\title{
AA-negative and Kappa-positive Amyloidosis in a Patient with Rheumatoid Arthritis
}

\author{
Toshiharu Ueno ${ }^{1}$, Keiichi Sumida ${ }^{1}$, Junichi Hoshino ${ }^{1}$, Tatsuya Suwabe ${ }^{1}$, Koki Mise ${ }^{1}$, \\ Ryo Hazue ${ }^{1}$, Noriko Hayami ${ }^{1}$, Rikako Hiramatsu ${ }^{1}$, Masahiro Kawada ${ }^{1}$, Aya Imafuku ${ }^{1}$, \\ Eiko Hasegawa ${ }^{1}$, Naoki Sawa ${ }^{1}$, Kenmei Takaichi ${ }^{1,2}$, Keiichi Kinowaki ${ }^{3}$, Kenichi Ohashi ${ }^{3}$, \\ Takeshi Fujii ${ }^{3}$, Aya Nishida ${ }^{4}$ and Yoshifumi Ubara ${ }^{1,2}$
}

\begin{abstract}
A 57-year-old Japanese woman with a 5-year history of rheumatoid arthritis (RA) was admitted to our hospital for an evaluation of nephrotic range proteinuria $(4.8 \mathrm{~g} / \mathrm{day})$. A renal biopsy led to the diagnosis of amyloidosis according to strong positivity for Congo red staining and the detection of microfibrillar structures on electron microscopy that were negative for AA and positive for kappa light chain. Combination therapy with high-dose melphalan and autologous stem cell transplantation was performed according to the regimen for $\mathrm{AL}$ amyloidosis. Her proteinuria and RA subsided, but relapsed after 3 years. This is the first report regarding kappa light chain amyloidosis in an RA patient.
\end{abstract}

Key words: kappa-positive amyloidosis, rheumatoid arthritis

(Intern Med 55: 2491-2495, 2016)

(DOI: 10.2169/internalmedicine.55.6796)

\section{Introduction}

When the coexistence of multiple myeloma is excluded, amyloidosis related to plasma cell dysplasia is referred to as primary amyloidosis, which includes AL-amyloidosis (ALAM) (due to an immunoglobulin light chain or fragment) and $\mathrm{AH}$ amyloidosis (due to truncated immunoglobulin heavy chains). Detection of a heavy chain or light chain monoclonal (M) protein in the serum or urine and positive kappa or lambda light chain staining of amyloid tissue are essential for the diagnosis of AL-AM. AL-AM is a systemic disease in which amyloid fibrils derived from incorrectlyproduced immunoglobulin form deposits that disrupt the function of various organs, including the kidneys, heart, and gastrointestinal tract $(1,2)$. Therefore, treatment of AL-AM targets the clonal bone marrow plasma cells using the methods employed for multiple myeloma, such as cyclic oral melphalan and prednisone therapy, melphalan with dexamethasone instead of prednisone, or high-dose intravenous melphalan followed by autologous stem cell transplantation (HDM/SCT) $(3,4)$. Recently, some novel agents for the treatment of plasma cell dyscrasias have been investigated. For instance, immunomodulators such as thalidomide and lenalidomide have been used in combination with dexamethasone and the proteasome inhibitor bortezomib has also shown efficacy $(2,5)$.

Amyloidosis can also be related to the chronic overproduction of the acute phase reactant protein serum amyloid A (SAA) protein in patients with chronic inflammatory diseases such as rheumatoid arthritis (RA) and familial Mediterranean fever. This is known as secondary AAamyloidosis, and positive staining of amyloid tissue for AA protein is essential for its diagnosis $(6,7)$. The AA amyloid fibril is a unique, non-immunoglobulin protein derived from SAA protein, a larger (12-kDa) serum precursor that is synthesized by the liver in response to cytokines such as interleukin-1 (IL-1), IL-6, and tumor necrosis factor-alpha (TNF-alpha) that are produced during chronic inflammation (1). Accordingly, primary therapy for AA-amyloidosis

${ }^{1}$ Nephrology Center, Toranomon Hospital Kajigaya, Japan, ${ }^{2}$ Okinaka Memorial Institute for Medical Research, Toranomon Hospital, Japan, ${ }^{3}$ Department of Pathology, Toranomon Hospital, Japan and ${ }^{4}$ Department of Hematology, Toranomon Hospital Kajigaya, Japan

Received for publication November 5, 2015; Accepted for publication December 8, 2015

Correspondence to Dr. Yoshifumi Ubara, ubara@toranomon.gr.jp 
includes biological agents (8-10).

We encountered a 57-year-old woman with RA and nephrotic range proteinuria. A renal biopsy led to a histological diagnosis of amyloidosis, however, she was negative for AA protein and positive for kappa light chain. Combination therapy with bortezomib and dexamethasone ameliorated her proteinuria, despite the use of biological agents for RA (TNF and IL-6 receptor analogues). This is the first report of AA-negative and kappa light chain-positive amyloidosis in a patient with RA.

\section{Case Report}

In August 2008, a 57-year-old Japanese woman was admitted to our hospital for an evaluation of nephrotic range proteinuria. In August 2003, she developed arthralgia of multiple joints, including the bilateral fingers, knees, and toes. Although an X-ray of her joints demonstrated minor abnormalities, antibody to cyclic citrullinated peptide, which is specific for a RA diagnosis, was serologically positive. According to these findings, RA had been diagnosed. Bucillamine was started two years after the diagnosis, after which methotrexate (MTX) was added at a dose of $8 \mathrm{mg}$ weekly with prednisolone at $5 \mathrm{mg}$ daily and treatment was continued until September 2008. Proteinuria (0.3 g daily) was detected in January 2007, and this progressed to $4.8 \mathrm{~g}$ daily by June 2008. She had no past history of diabetes, hypertension, or dyslipidemia.

On admission, the patient was $158 \mathrm{~cm}$ tall and weighed $53 \mathrm{~kg}$. Her temperature was $36.8^{\circ} \mathrm{C}$, pulse rate was $60 / \mathrm{min}$, and blood pressure was 98/68 mmHg. Her hands, knees, feet, and ankles were swollen and tender bilaterally, and there was bilateral pitting edema of the lower extremities. A blood count included hemoglobin of $12.1 \mathrm{~g} / \mathrm{dL}$, white blood cells of $7,600 / \mu \mathrm{L}$, platelet count of $36.0 \times 10^{4} / \mu \mathrm{L}$, which remained nearly unchanged throughout her clinical course. Laboratory findings included serum albumin of $2.2 \mathrm{~g} / \mathrm{dL}$, total protein of $4.5 \mathrm{~g} / \mathrm{dL}$, urea nitrogen of $13.2 \mathrm{mg} / \mathrm{dL}$, and creatinine of $0.58 \mathrm{mg} / \mathrm{dL}$. Immunological tests revealed that antinuclear antibody was negative, anti-cyclic citrullinated peptide (CCP) antibody was $1,570 \mathrm{ng} / \mathrm{mL}$ (normal <4.5), rheumatoid factor (RF) was $6 \mathrm{U} / \mathrm{mL}$ (normal <10), Creactive protein (CRP) was $2.3 \mathrm{mg} / \mathrm{dL}$, and the erythrocyte sedimentation rate (ESR) was $86 \mathrm{~mm} / \mathrm{h}$. SSA was $24.5 \mu \mathrm{g} /$ $\mathrm{mL}$ (normal: <8.0). CH50 was $82 \mathrm{U} / \mathrm{mL}$ (normal >30 U/ $\mathrm{mL})$, serum immunoglobulin $\mathrm{G}(\mathrm{IgG})$ was $768 \mathrm{mg} / \mathrm{dL}$, IgA was $193 \mathrm{mg} / \mathrm{dL}$, and $\operatorname{IgM}$ was $135 \mathrm{mg} / \mathrm{dL}$. An examination of free light chains disclosed a serum $\kappa$ light chain level of 2.26 and a $\lambda$ light chain level of $4.29 \mathrm{mg} / \mathrm{dL}$, with a $\kappa / \lambda$ ratio of 0.53 (normal: 0.3 to 1.6). Serum M protein (immunofixation electrophoresis) and cryoglobulin were negative. Urinary protein excretion was $4.8 \mathrm{~g} /$ day, and the sediment contained 6 to 10 erythrocytes per high-power field (HPF). Urinary Bence Jones protein (immunofixation electrophoresis) was negative. The disease activity score (DAS)CRP score was calculated to be 4.5 .
A gastroenterological endoscopic and colonoscopic biopsy was performed for the detection of amyloid deposition in the intestinal mucosa. The samples showed amyloid deposition in the walls of the subepithelial vessels by Direct Fast Scarlet (DFS) staining. Bone marrow aspiration revealed slightly hyperplastic bone marrow with amyloid deposition by DFS staining. Although slightly hyperplastic plasma cells were observed, they were less than $10 \%$ cellularity and did not fit the criteria for multiple myeloma. Granulocytic differentiation was preserved, and other abnormal findings were not observed on the blood smear. No osteolytic lesions were detected by a full skeletal survey. These findings did not fit the criteria for multiple myeloma or monoclonal gammopathy of undetermined significance (MGUS).

\section{Renal biopsy findings}

A light microscopic examination of a renal biopsy specimen containing 30 glomeruli showed global sclerosis in only 1 glomerulus. Nearly all remaining glomeruli demonstrated mesangial expansion by homogenous and acellular amorphous deposits that did not contain either cellular matrix or collagen on periodic acid methenamine silver (PAM) staining (Fig. 1a). These deposits were strongly positive for Congo red staining (Fig. 1b). Similar deposits were also observed in the walls of small arteries, such as the glomerular vasculature, as well as arterioles and interlobular arteries. Immunofluorescence for $\operatorname{IgG}, \operatorname{Ig} \mathrm{A}, \operatorname{IgM}$, and C3 was negative. We pretreated the sections with a solution of potassium permanganate $\left(\mathrm{KMnO}_{4}\right)$ prior to staining with Congo red; however, the procedure demonstrated no decrease of intensity in amyloid deposition, suggesting that the type of amyloidosis in this patient to be the non-AA type. Electron microscopy revealed randomly arranged microfibrils measuring 8-12 $\mathrm{nm}$ in diameter in the expanded mesangial areas (Fig. 1c and inset of Fig. 1c). Immunofluorescence of serial sections demonstrated that the deposits were predominantly positive for kappa light chain (Fig. 1d), rather than for lambda chain (Fig. 1e), but negative for anti-AA antibody (Fig. 1f). These findings confirmed the diagnosis of AAnegative and kappa-positive renal amyloidosis.

\section{Clinical course}

The patient's clinical course is shown in Fig. 2. After the renal biopsy, prednisolone and MTX were discontinued. Based on the typical treatment for AL-amyloidosis, autologous stem cell transplantation (SCT) was performed according to the previous criteria and regimen $(11,12)$. SCT was performed after she received 2 cycles of chemotherapy with vincristine, daunorubicin, dexamethasone (VAD) and highdose melphalan (HDM). Her arthralgia subsided immediately after SCT and the RA disease activity became intact with DAS-CRP of 0.96 for two years. Proteinuria gradually declined toward $3.0 \mathrm{~g}$ daily within one year after SCT and decreased to $1.0 \mathrm{~g}$ daily by August 2011. However, relapse of polyarthralgia affecting the bilateral knees, hands, and feet occurred in June 2011 with an elevation of the CRP 

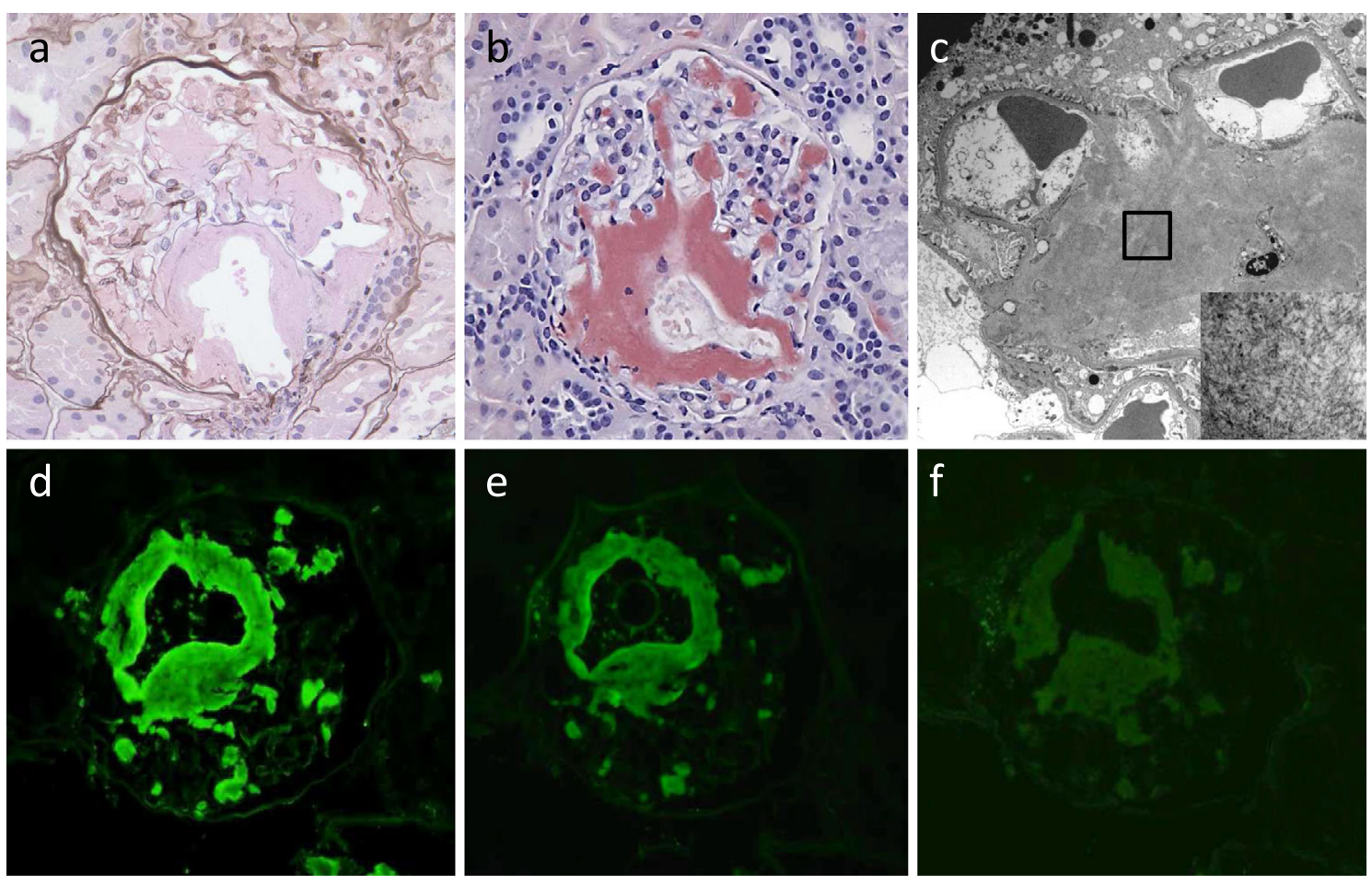

Figure 1. Renal biopsy. a: Mesangial areas are expanded by homogenous and acellular amorphous deposits that are negative for cell matrix or collagen on PAM staining. b: These deposits are strongly positive for Congo red staining. c: Electron microscopy reveals amorphous mesangial electron-dense deposits (original magnification, 4,500x), and the dense deposits show randomly arranged microfibrils measuring 8-12 $\mathrm{nm}$ in diameter (inset, original magnification 9,000x). $d$ and e: The deposits are predominantly positive for kappa light chain (Fig. 1d), rather than for lambda chain (Fig. 1e). f: The deposits are negative for anti-AA antibody. All primary antibodies for immunofluorescence were purchased from Dako (Glostrup, Denmark); kappa (A-0191), lambda (A-0193), and AA (M-0759).

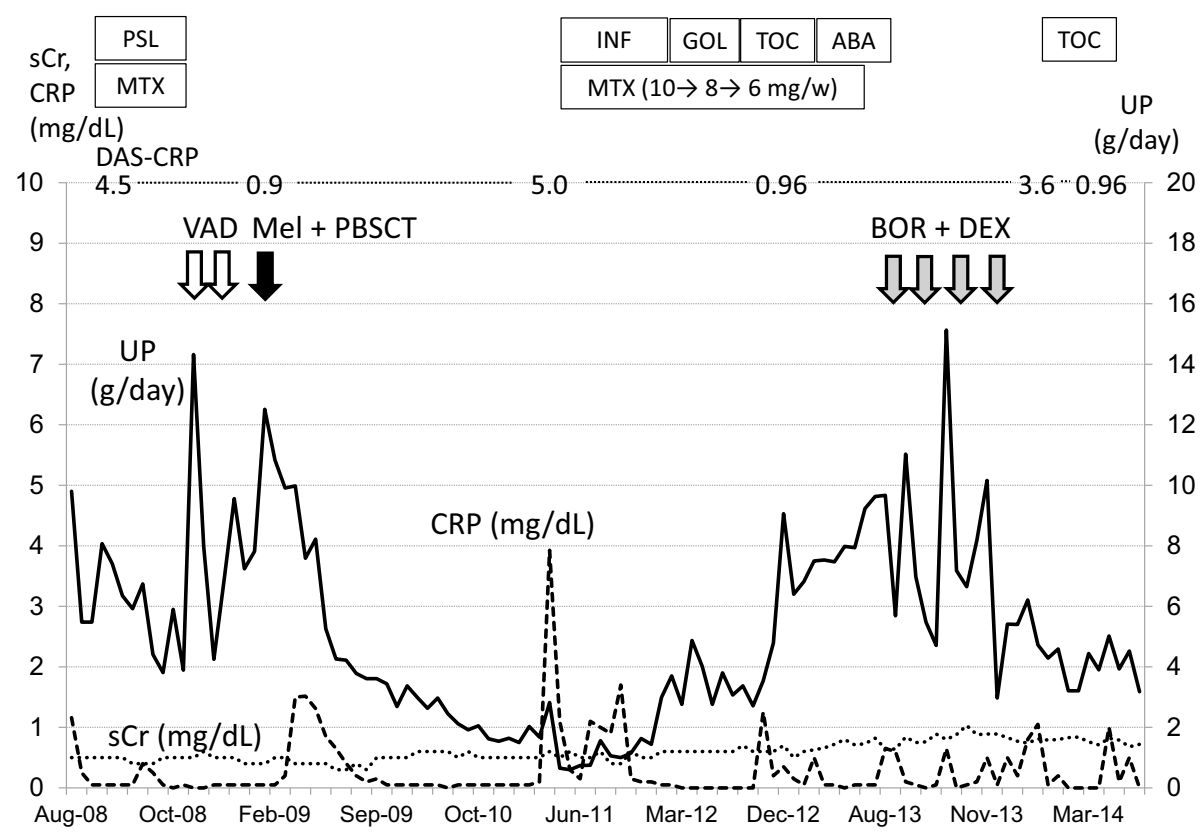

Figure 2. Clinical course. PSL: prednisolone, MTX: methotrexate, INF: infliximab, GOL: golimumab, TOC: tocilizumab, ABA: abatacept, VAD: vincristine, Adriamycin (daunorubicin), and dexamethasone, HDM: high-dose melphalan, PBSCT: peripheral blood stem cell transplantation, BOR: bortezomib, DEX: dexamethasone 
level to $3.4 \mathrm{mg} / \mathrm{dL}$ (DAS-CRP of 5.0) and the ESR to 91 $\mathrm{mm} / \mathrm{h}$. Combination therapy with infliximab $(3 \mathrm{mg} / \mathrm{kg})$ and MTX (10 mg weekly) was started, however, her RA did not subside. The biological agent was switched to golimumab (50 mg), but her RA remained unchanged. Next tocilizumab (8 $\mathrm{mg} / \mathrm{kg}$ ) was started. RA subsided to DAS-CRP of 0.96 , but tocilizumab was discontinued due to a decrease in the IgG value and switched to abatacept (125 mg weekly); the RA disease activity remained stable. However, proteinuria increased to $11 \mathrm{~g}$ daily in July 2013. An examination of free light chains disclosed a serum $\kappa$ light chain level of 26.4 and a $\lambda$ light chain level of $12.9 \mathrm{mg} / \mathrm{dL}$, with a $\kappa / \lambda$ ratio of 2.05. Serum M protein (immunofixation electrophoresis) and cryoglobulin were still negative. We performed a repeat biopsy of the kidney and colon before treatment with bortezomib. A re-biopsy of the kidney revealed a more severe accumulation of amyloid deposits in both the mesangial area and glomerular capillary wall, and it also showed that this amyloidosis was kappa-positive and AA-negative, similar to the first renal biopsy. A colonoscopic re-biopsy showed nearly the same degree of amyloid deposition. Combination therapy was started with bortezomib (1.95 mg/day) and dexamethasone (20 mg/day). The bortezomib-dexamethasone combination therapy was performed once a month and repeated 4 times. The effect of reduced proteinuria persisted for six months from the last administration. Her RA remained subsided and proteinuria decreased to $3 \mathrm{~g}$ daily in March 2014, however, the RA disease activity subsequently relapsed to DAS-CRP of 3.6 and tocilizumab was started again, and RA subsided to DAS-CRP of 0.96. Her proteinuria remained at a low level, regardless of the RA activity.

\section{Discussion}

Although HDM/SCT has been attempted as treatment for AL-AM, Gertz previously reported the limitations of this approach. Patients with AL-AM who have advanced amyloid cardiomyopathy or the involvement of more than 2 major organs are poor candidates for SCT, while patients with 1-2 organ involvement or early cardiac changes are usually more appropriate candidates (2). Leung et al. reported that HDM/ SCT is the most effective treatment for AL-AM if the patient is eligible. A renal response was achieved in $60.3 \%$ of all patients evaluated, with proteinuria being reduced by more than $90 \%$ in $37.9 \%$ of the patients and normalized in $15.5 \%$ of the patients. They also reported that renal responders had a better survival regardless of their hematologic response (4). Kastritis et al. proposed the use of combination therapy with bortezomib and dexamethasone (BD) for ALAM and they treated 18 patients with BD, including seven who had relapsed or progressed on previous therapies. They found a rapid hematologic response and stated that toxicity could be managed with a careful follow-up and appropriate dose adjustment. They also stated that this treatment might be a valid option for patients with severe heart or kidney impairment (5). This report supports the use of BD therapy as a therapeutic option for our patient with amyloidosis.

Okuda et al. reported that tocilizumab was effective in a patient with progressive AA amyloidosis complicating active juvenile idiopathic arthritis that was resistant to other therapies, and they confirmed the marked regression of AAamyloid deposits by ab endoscopic mucosal biopsy of the gastrointestinal tract (8). Hattori et al. reported that hemodialysis patients with AA amyloidosis showed an improvement of myocardial hypertrophy and dialysis hypotension, as well as a histological improvement of amyloid deposits in the gastrointestinal tract, after tocilizumab therapy (9). If the present patient's amyloidosis was AA type, then her proteinuria and activity of RA must be paralleled and her arthralgia and SAA level would be closely associated. However, her proteinuria relapsed independently of the RA activity. Thus, her amyloidosis was not AA-type associated with RA.

The efficacy of SCT for RA is considered to be due to the reeducation of hematopoietic stem cell-derived lymphocytes after ablation of self-reactive lymphocytes using chemotherapy. In 2004, Snowden et al. reported the use of autologous SCT to treat severe RA. To perform SCT, healthy stem cells are amplified using granulocyte-colony stimulating factor (G-CSF) and are re-injected into patients with the expectation of improving the bone marrow function. Autologous SCT is a relatively safe salvage treatment for severe treatment-resistant RA. In these open-label studies, significant responses were achieved in most patients, with over $50 \%$ achieving ACR 50 or better at 12 months (13). However, Tyndall et al. reported that although performing autologous SCT for RA achieved a positive short-term outcome with low treatment-related toxicity, most patients subsequently relapsed (14). The premedication of SCT is completely different between SCT for treatment-resistant connective tissue diseases and SCT for AL-amyloidosis combined with chemotherapy; the former uses cyclophosphamide (CYC) 2 to $4 \mathrm{~g} / \mathrm{m}^{2}$ and/or etoposide $300 \mathrm{mg} / \mathrm{m}^{2}$, and the latter uses high-dose melphalan. This difference might contribute to the differences observed after the therapeutic prognosis. The present patient's RA remained subsided and proteinuria decreased to $3 \mathrm{~g}$ daily in March 2014, however, RA subsequently relapsed to DAS-CRP of 3.6 and tocilizumab was started again, and RA subsided to DASCRP of 0.96. Her proteinuria remained at a low level, regardless of the RA activity.

In this case, a renal biopsy disclosed AA-negative and kappa-positive amyloidosis.

Biological agents for RA relapse were subsequently administered and the patient's RA subsided, however, it did not respond to nephrotic range proteinuria. Proteinuria and the RA activity were not associated, and the combination of VAD plus HDM/SCT or the combination of BD therapy drastically ameliorated her proteinuria.

The clinical course of this patient indicated that kappa light chain amyloidosis was not secondary to the overproduction of cytokines derived from RA, but was more related to a mechanism mediated by bone marrow stem cells. 
This patient provided her informed consent for the publication of this case report.

The authors state that they have no Conflict of Interest (COI).

\section{Financial Support}

This study was funded by the Okinaka Memorial Institute for Medical Research.

\section{References}

1. Maitra A. Amyloidosis. In: Robins’ Basic Patology. 9th ed. Kumar V, Abbas AK, Aster JC, Eds. Elsevier Saunders, Philadelphia, 2013: 153-159.

2. Gertz MA. Immunoglobulin light chain amyloidosis: 2013 update on diagnosis, prognosis, and treatment. Am J Hematol 88: 416425, 2013.

3. Comenzo RL, Gertz MA. Autologous stem cell transplantation for primary systemic amyloidosis. Blood 99: 4276-4282, 2002.

4. Leung N, Dispenzieri A, Fervenza FC, et al. Renal response after high-dose melphalan and stem cell transplantation is a favorable marker in patients with primary systemic amyloidosis. Am J Kidney Dis 46: 270-277, 2005.

5. Kastritis E, Anagnostopoulos A, Roussou M, et al. Treatment of light chain (AL) amyloidosis with the combination of bortezomib and dexamethasone. Haematologica 92: 1351-1358, 2007.

6. Bergesio F, Ciciani AM, Manganaro M, et al; Renal involvement in systemic amyloidosis, an Italian collaborative study on survival and renal outcome. Immunopathology Group of the Italian Society of Nephrology. Nephrol Dial Transplant 23: 941-951, 2008.
7. Lachmann HJ, Goodman HJ, Gilbertson JA, et al. Natural history and outcome in systemic AA amyloidosis. $N$ Engl J Med 356: 2361-2371, 2007.

8. Okuda Y, Takasugi K. Successful use of a humanized antiinterleukin-6 receptor antibody, tocilizumab, to treat amyloid A amyloidosis complicating juvenile idiopathic arthritis. Arthritis Rheum 54: 2997-3000, 2006.

9. Hattori Y, Ubara Y, Sumida K, et al. Tocilizumab improves cardiac disease in a hemodialysis patient with AA amyloidosis secondary to rheumatoid arthritis. Amyloid 19: 37-40, 2012.

10. Nakamura A, Matsuda M, Tazawa K, Shimojima Y, Ikeda S. Successful treatment with infliximab and low-dose methotrexate in a Japanese patient with familial Mediterranean fever. Intern Med 46: 1247-1249, 2007.

11. Yamazaki O, Ubara Y, Suwabe T, et al. Successful treatment of primary AL amyloidosis by VAD therapy, high-dose melphalan, and autologous peripheral stem cell transplantation. Clin Exp Nephrol 13: 522-525, 2009.

12. Sakurai-Chin C, Ubara Y, Suwabe T, et al. AL amyloidosis with IgD-lambda monoclonal gammopathy and lambda-type BenceJones protein: successful treatment by autologous stem cell transplantation. Clin Exp Nephrol 14: 506-510, 2010.

13. Snowden JA, Passweg J, Moore JJ, et al. Autologous hemopoietic stem cell transplantation in severe rheumatoid arthritis: a report from the EBMT and ABMTR. J Rheumatol 31: 482-488, 2004.

14. Tyndall A, van Laar JM. Stem cells in the treatment of inflammatory arthritis. Best Pract Res Clin Rheumatol 24: 565-574, 2010.

The Internal Medicine is an Open Access article distributed under the Creative Commons Attribution-NonCommercial-NoDerivatives 4.0 International License. To view the details of this license, please visit (https://creativecommons.org/licenses/ by-nc-nd/4.0/).

(C) 2016 The Japanese Society of Internal Medicine http://www.naika.or.jp/imonline/index.html 